\title{
A THEOREM OF J. L. WALSH, REVISITED
}

\author{
A. S. Cavaretta, Jr., A. Sharma, and R. S. Varga \\ Dedicated to the memory of Ernst G. Straus
}

The well-known and beautiful result of $J$. L. Walsh, on the overconvergence of sequences of differences of polynomials interpolating a function $f(z)$ analytic in $|z|<\rho$ (but having a singularity on $|z|=\rho$ ), where $1<\rho<\infty$, has been recently extended in a new direction by T. J. Rivlin. We give here three new extensions of Rivlin's result, which include Hermite and Birkhoff interpolation.

1. Introduction. Let $A_{\rho}$ denote the collection of functions analytic in $|z|<\rho$ and having a singularity on the circle $|z|=\rho$ (where we assume throughout that $1<\rho<\infty)$. For each $f(z)=\sum_{k=0}^{\infty} a_{k} z^{k}$ in $A_{\rho}$ and for each positive integer $n$, let

$$
s_{n}(z ; f):=\sum_{k=0}^{n} a_{k} z^{k}
$$

be the $n$th partial sum of $f(z)$, and let $L_{n}(z ; f)$ similarly denote the unique Lagrange interpolation polynomial (of degree at most $n$ ) which interpolates $f(z)$ in the $(n+1)$-st roots of unity, i.e., if $\omega$ is a primitive root of $\omega^{n+1}=1$,

$$
L_{n}\left(\omega^{k} ; f\right)=f\left(\omega^{k}\right), \quad \text { for all } k=0,1,2, \ldots, n .
$$

Then, a well-known and beautiful result of J. L. Walsh [8, p. 153] can be stated as

TheOREM A. ([8]). For each $f \in A_{\rho}$, there holds

$$
\lim _{n \rightarrow \infty}\left\{L_{n}(z ; f)-s_{n}(z ; f)\right\}=0, \text { for all }|z|<\rho^{2},
$$

the convergence being uniform and geometric on any closed subset of $|z|<\rho^{2}$. More precisely, for any $\tau$ with $\rho \leq \tau<\infty$, there holds

$$
\varlimsup_{n \rightarrow \infty}\left\{\max _{|z| \leq \tau}\left|L_{n}(z ; f)-s_{n}(z ; f)\right|\right\}^{1 / n} \leq \frac{\tau}{\rho^{2}} .
$$

Further, the result of (1.3) is best possible in the sense that there is some $\hat{f} \in A_{\rho}$ and some $\hat{z}$ with $|\hat{z}|=\rho^{2}$ for which the sequence

$$
\left\{L_{n}(\hat{z} ; \hat{f})-s_{n}(\hat{z} ; \hat{f})\right\}_{n=1}^{\infty}
$$

does not tend to zero as $n \rightarrow \infty$. 
For general discussions of various extensions of Walsh's Theorem A, see for example [2] and [7]. Recently, Rivlin [4] has obtained some interesting new analogues of Walsh's Theorem. Here, we shall show that one of Rivlin's results [4, Theorem 1] can be further generalized. In order to describe these extensions, we introduce some needed notation.

First, let $\pi_{k}$ as usual denote the collection of all complex polynomials of degree at most $k$. Next, consider all positive integers $m$ of the form $m=q n+c$ where $q$ and $c$ are fixed positive integers, so that $m \geq n+1$. With $\omega$ a primitive $m$ th root of unity, and with $r$ a fixed nonnegative integer, we propose to find, for each $f \in A_{\rho}$, the polynomial $P_{r m+n}(z ; f)$ in $\pi_{r m+n}$ which satisfies the Hermite interpolation conditions

$$
\begin{aligned}
& P_{r m+n}^{(\nu)}\left(\omega^{k} ; f\right)=f^{(\nu)}\left(\omega^{k}\right), \\
& \quad \text { for all } k=0,1, \ldots, m-1 ; \nu=0,1, \ldots, r-1, \quad \text { if } r \geq 1,
\end{aligned}
$$

and which also minimizes

$$
\sum_{k=0}^{m-1}\left|P_{r m+n}^{(r)}\left(\omega^{k} ; f\right)-f^{(r)}\left(\omega^{k}\right)\right|^{2}
$$

over all polynomials in $\pi_{r m+n}$ which satisfy the interpolation conditions of (1.5). (The existence and uniqueness of this polynomial $P_{r m+n}(z ; f)$, while a basic consequence of approximation theory, will follow from the explicit representations of (2.4) and (2.8) in §2.)

In $\S 2$, we study the difference

$$
P_{r m+n}(z ; f)-s_{r m+n}(z ; f)
$$

in Theorem 1, and show that it tends to zero, as $n \rightarrow \infty$ in

$$
|z|<\rho^{1+q /(1+r q)}
$$

thereby extending Rivlin's result [4, Theorem 1]. In $\S 3$, we state extensions of Theorem 1 to Birkhoff interpolation, in which the Hermite interpolation condition of (1.5) is replaced by more general Birkhoff interpolation conditions (cf. (3.2)).

\section{An Extension of Rivlin's Result. We first establish}

THEOREM 1. For each $f \in A_{\rho}$ and for each nonnegative integer $r$, let the polynomials $P_{r m+n}(z ; f)$ and $s_{r m+n}(z ; f)$ be defined as in $(1.5)-(1.6)$ and (1.1). With $m=n q+c$, where $q$ and $c$ are any fixed positive integers, there holds

$$
\lim _{n \rightarrow \infty}\left\{P_{r m+n}(z ; f)-s_{r m+n}(z ; f)\right\}=0, \text { for all }|z|<\rho^{1+q /(1+r q)}
$$


the convergence being uniform and geometric on any closed subset of $|z|<\rho^{1+q /(1+r q)}$. More precisely, for any $\tau$ with $\rho<\tau<\infty$, there holds

$$
\varlimsup_{n \rightarrow \infty}\left\{\max _{|z| \leq \tau}\left|P_{r m+n}(z ; f)-s_{r m+n}(z ; f)\right|\right\}^{1 / n} \leq \frac{\tau^{r q+1}}{\rho^{(r+1) q+1}} .
$$

Further, the result of (2.1) is best possible in the sense that there is some $\hat{f} \in A_{\rho}$ and some $\hat{z}$ with $|\hat{z}|=\rho^{1+q /(1+r q)}$ for which the sequence $\left\{P_{r m+n}(\hat{z} ; \hat{f})-s_{r m+n}(\hat{z} ; \hat{f})\right\}_{n=1}^{\infty}$ does not tend to zero as $n \rightarrow \infty$.

We remark that as the special case $r=0$ of Theorem 1 reduces to Rivlin's result [4, Theorem 1], then the above result generalizes Rivlin's result.

To begin, for each $f \in A_{\rho}$, let $h_{r m-1}(z ; f)$ be the unique Hermite interpolation polynomial of $f(z)$ in $\pi_{r m-1}$ which satisfies (1.5), i.e.,

$$
h_{r m-1}^{(\nu)}\left(\omega^{k} ; f\right)=f^{(\nu)}\left(\omega^{k}\right)
$$

for all $k=0,1, \ldots, m-1, \nu=0,1, \ldots, r-1$, if $r \geq 1$; otherwise, $h_{r m-1}(z ; f) \equiv 0$ if $r=0$. Then, any $P_{r m+n}(z ; f)$ satisfying (1.5) necessarily has the form

$$
P_{r m+n}(z ; f)=h_{r m-1}(z ; f)-\left(z^{m}-1\right)^{r} Q_{n}(z),
$$

where $Q_{n} \in \pi_{n}$. Since

$$
\left.\frac{d^{r}}{d z^{r}}\left(z^{m}-1\right)^{r}\right|_{z=\omega^{k}}=\omega^{-k r} \sum_{\nu=0}^{r}(-1)^{r-\nu}\left(\begin{array}{l}
r \\
\nu
\end{array}\right)(m \nu)_{r}=\omega^{-k r} m^{r} r !
$$

(where $(x)_{0}:=1$ and where $(x)_{k}:=x(x-1) \cdots(x-k+1)$ when $k$ is a positive integer), it easily follows from (2.4) that the problem of minimizing (1.6) is equivalent to finding the polynomial $Q_{n}(z)$ in $\pi_{n}$ which solves

$$
\sum_{k=0}^{m-1}\left|g\left(\omega^{k}\right)-Q_{n}\left(\omega^{k}\right)\right|^{2}=\min _{p_{n} \in \pi_{n}} \sum_{k=0}^{m-1}\left|g\left(\omega^{k}\right)-p_{n}\left(\omega^{k}\right)\right|^{2},
$$

where

$$
g(z):=z^{r}\left\{h_{r m-1}^{(r)}(z ; f)-f^{(r)}(z)\right\} /\left[m^{r} \cdot r !\right] .
$$

We next establish

LEMMA 1. The polynomial $Q_{n}(z)$ in $\pi_{n}$ which solves (2.6) is explicitly given by

$$
Q_{n}(z)=-\frac{1}{2 \pi i} \int_{\Gamma} \frac{f(t) t^{m-n-1}\left(t^{n+1}-z^{n+1}\right) d t}{(t-z)\left(t^{m}-1\right)^{r+1}},
$$

where $\Gamma:=\{z:|z|=R\}$ and where $R$ is any number satisfying $1<R<\rho$. 
Proof. By Hermite's interpolation formula (cf. [2, p. 164]), we know that the polynomials $h_{r m-1}(z ; f)$ of $(2.3)$ and $s_{r m+n}(z ; f)$ of $(1.1)$ can be expressed as

$$
\left\{\begin{array}{l}
h_{r m-1}(z ; f)=\frac{1}{2 \pi i} \int_{\Gamma} \frac{f(t)\left[\left(t^{m}-1\right)^{r}-\left(z^{m}-1\right)^{r}\right] d t}{(t-z)\left(t^{m}-1\right)^{r}}, \text { and } \\
s_{r m+n}(z ; f)=\frac{1}{2 \pi i} \int_{\Gamma} \frac{f(t)\left[t^{r m+n+1}-z^{r m+n+1}\right] d t}{(t-z) t^{r m+n+1}}
\end{array}\right.
$$

Thus, from Cauchy's integral formula, we can write

$$
h_{r m-1}(z ; f)-f(z)=-\frac{1}{2 \pi i} \int_{\Gamma} \frac{f(t) K(t, z) d t}{\left(t^{m}-1\right)^{r}},
$$

where

$$
K(t, z):=\frac{\left(z^{m}-1\right)^{r}}{t-z} .
$$

From (2.5), we see that

$$
\left.z^{r} \frac{\partial^{r}}{\partial z^{r}} K(t, z)\right|_{z=\omega^{k}}=\frac{m^{r} \cdot r !}{t-\omega^{k}}, \quad k=0,1, \ldots, m-1 .
$$

Thus, on differentiating $r$ times with respect to $z$ in (2.10) and using (2.12), it follows that the Lagrange polynomial interpolant $L_{m-1}(z ; g)$ of (1.2) of $g(z)$ (defined in (2.7)) in the points $\omega^{k}, k=0,1, \ldots, m-1$, is just

$$
L_{m-1}(z ; g)=-\frac{1}{2 \pi i} \int_{\Gamma} \frac{f(t)\left(t^{m}-z^{m}\right) d t}{(t-z)\left(t^{m}-1\right)^{r+1}},
$$

from which it follows (cf. (1.1)) that

$$
s_{n}\left(z ; L_{m-1}(z ; g)\right)=-\frac{1}{2 \pi i} \int_{\Gamma} \frac{f(t) t^{m-n-1}\left(t^{n+1}-z^{n+1}\right) d t}{(t-z)\left(t^{m}-1\right)^{r+1}}
$$

But, Rivlin [4] has shown that the solution $Q_{n}(z)$ of (2.6) satisfies $Q_{n}(z)=s_{n}\left(z ; L_{m-1}(z ; g)\right)$, so that (2.13) gives the desired integral representation for $Q_{n}(z)$ in (2.8).

This brings us to the

Proof of Theorem 1. From (2.4), (2.8), and (2.9), we can write

$$
P_{r m+n}(z ; f)-s_{r m+n}(z ; f)=\frac{1}{2 \pi i} \int_{\Gamma} \frac{f(t) K_{1}(t, z) d t}{(t-z)},
$$


where

$$
K_{1}(t, z):=\frac{z^{r m+n+1}}{t^{r m+n+1}}+\left(\frac{z^{m}-1}{t^{m}-1}\right)^{r}\left\{\frac{1-t^{m-n-1} z^{n+1}}{t^{m}-1}\right\} .
$$

Next, set (cf. [2, p. 163])

$$
\beta_{j}\left(z^{m}\right)=\beta_{j}\left(z^{m} ; r\right):=\sum_{k=0}^{r-1}\left(\begin{array}{c}
r+j-1 \\
k
\end{array}\right)\left(z^{m}-1\right)^{k},
$$$$
\text { for all } j=1,2, \ldots \text {, }
$$

so that $\beta_{j}\left(z^{m}\right)$ is in $\pi_{(r-1) m}$ for each $j \geq 1$. Moreover, the following identity holds (cf. [2]):

$$
\left(\frac{z^{m}-1}{t^{m}-1}\right)^{r}=\frac{z^{r m}}{t^{r m}}-\frac{\left(t^{m}-z^{m}\right)}{t^{(r+1) m}} \sum_{s=0}^{\infty} \frac{\beta_{s+1}\left(z^{m}\right)}{t^{s m}} .
$$

We note from (2.16) that

$$
\left|\beta_{j}\left(z^{m}\right)\right| \leq 2^{r+j-1}\left(|z|^{m}+1\right)^{r-1} \text { for all } j \geq 1,
$$

so that the last sum in (2.17) converges absolutely for any $t$ with $|t|>1$, provided that $m$ is sufficiently large. Inserting the identity of (2.17) in (2.15), it readily follows that $K_{1}(t, z)$ can be expressed as the sum

$$
K_{1}(t, z)=T_{1}(t, z)+T_{2}(t, z)+T_{3}(t, z)
$$

where

$$
\left\{\begin{array}{l}
T_{1}(t, z):=\frac{z^{r m}\left(t^{n+1}-z^{n+1}\right)}{t^{(r+1) m+n+1}} \sum_{s=0}^{\infty} \frac{1}{t^{s m}} \\
T_{2}(t, z):=\frac{z^{n+1}\left(t^{m}-z^{m}\right)}{t^{(r+1) m+n+1}} \sum_{s=0}^{\infty} \frac{\gamma_{s}\left(z^{m}\right)}{t^{s m}} \\
T_{3}(t, z):=-\frac{\left(t^{m}-z^{m}\right)}{t^{(t+2) m}} \sum_{s=0}^{\infty} \frac{\gamma_{s}\left(z^{m}\right)}{t^{s m}}
\end{array}\right.
$$

and where

$$
\gamma_{s}\left(z^{m}\right):=\sum_{j=0}^{s} \beta_{j+1}\left(z^{m}\right), \text { for all } s=0,1, \ldots,
$$

so that $\gamma_{s}\left(z^{m}\right)$ is in $\pi_{(r-1) m}$ for all $s \geq 0$.

If $\max _{|t|=R}|f(t)|=: M_{R}$, then for $|z|=\tau \geq \rho$ and for $|t|=R$ where $1<R<\rho$, we have

$$
\begin{aligned}
& \left|\frac{1}{2 \pi i} \int_{\Gamma} \frac{f(t) T_{2}(t, z) d t}{t-z}\right| \\
& \quad \leq \frac{M_{R} \tau^{n+1}\left(R^{m}+\tau^{m}\right)}{(\tau-R) R^{(r+1) m+n}}\left\{\left|\gamma_{0}\left(z^{m}\right)\right|+\frac{\left|\gamma_{1}\left(z^{m}\right)\right|}{R^{m}}+\cdots\right\} .
\end{aligned}
$$


As $\left|\gamma_{0}\left(z^{m}\right)\right|=\left|\beta_{1}\left(z^{m}\right)\right| \leq 2^{r} \tau^{(r-1) m}\left(1+1 / \tau^{m}\right)^{r-1}$ from (2.21) and (2.18), an easy calculation shows, after recalling that $m=n q+c$, that

$$
\varlimsup_{n \rightarrow \infty}\left\{\max _{|z| \leq \tau}\left|\frac{1}{2 \pi i} \int_{\Gamma} \frac{f(t) T_{2}(t, z) d t}{t-z}\right|\right\}^{1 / n} \leq \frac{\tau^{r q+1}}{R^{(r+1) q+1}} .
$$

But, as the left side of (2.22) is independent of the choice of $R$ with $1<R<\rho$, we see that

$$
\varlimsup_{n \rightarrow \infty}\left\{\max _{|z| \leq \tau}\left|\frac{1}{2 \pi i} \int_{\Gamma} \frac{f(t) T_{2}(t, z) d t}{t-z}\right|\right\}^{1 / n} \leq \frac{\tau^{r q+1}}{\rho^{(r+1) q+1}},
$$

A similar calculation gives that

$$
\varlimsup_{n \rightarrow \infty}\left\{\max _{|z| \leq \tau}\left|\frac{1}{2 \pi i} \int_{\Gamma} \frac{f(t) T_{1}(t, z) d t}{t-z}\right|\right\}^{1 / n} \leq \frac{\tau^{r q+1}}{\rho^{(r+1) q+1}},
$$

and

$$
\varlimsup_{n \rightarrow \infty}\left\{\max _{|z| \leq \tau}\left|\frac{1}{2 \pi i} \int_{\Gamma} \frac{f(t) T_{3}(t, z) d t}{t-z}\right|\right\}^{1 / n} \leq \frac{\tau^{r q}}{\rho^{(r+2) q}} .
$$

Since $\tau^{r q} / \rho^{(r+2) q} \leq \tau^{r q+1} / \rho^{(r+1) q+1}$, it follows from (2.14), (2.19), and (2.23)-(2.25) that

$$
\varlimsup_{n \rightarrow \infty}\left\{\max _{|z| \leq \tau}\left|P_{r m+n}(z ; f)-s_{r m+n}(z ; f)\right|\right\}^{1 / n} \leq \frac{\tau^{r q+1}}{\rho^{(r+1) q+1}},
$$

for any $\tau$ with $\rho \leq \tau<\infty$, which establishes both (2.1) and (2.2) of Theorem 1.

Finally, to establish the sharpness of (2.1) in Theorem 1, it suffices to take $\hat{f}(z):=(\rho-z)^{-1}$ and $\hat{z}=\rho^{1+q /(1+r q)}$, which was also in essence used by Walsh [8, p. 154] to establish the sharpness of (1.3) of his Theorem A. Omitting the calculations, we simply state that

$$
\lim _{n \rightarrow \infty}\left\{P_{r m+n}(\hat{z}: \hat{f})-s_{r m+n}(\hat{z}: \hat{f})\right\}=\frac{(1+r) \rho^{\beta}}{\rho^{\alpha}-\rho} \neq 0,
$$

where

$$
\alpha:=1+\frac{q}{1+r q}, \quad \beta:=\frac{q-c}{1+r q},
$$

which yields the desired sharpness of (2.1) of Theorem 1.

To motivate our next result, consider any $f(z)=\sum_{j=0}^{\infty} a_{j} z^{j}$ in $A_{\rho}$, and set

$$
s_{n, j}(z ; f):=\sum_{k=0}^{n} a_{k+j(n+1)} z^{k}, \quad j=0,1, \ldots,
$$


so that $s_{n, j}(z ; f) \in \pi_{n}$ for each $j \geq 0$. Moreover, we see from (1.1) that $s_{n}(z ; f)=s_{n, 0}(z ; f)$. The following known result gives Walsh's Theorem A as the special case $l=1$.

THEOREM B ([2]). For each $f \in A_{\rho}$ and for each positive integer $l$, there holds

(2.28) $\lim _{n \rightarrow \infty}\left\{L_{n}(z ; f)-\sum_{j=0}^{l-1} s_{n, j}(z ; f)\right\}=0 \quad$ for all $|z|<\rho^{l+1}$,

the convergence being uniform and geometric on any closed subset of $|z|<\rho^{l+1}$. More precisely, for any $\tau$ with $\rho \leq \tau<\infty$, there holds

$$
\varlimsup_{n \rightarrow \infty}\left\{\max _{|z| \leq \tau}\left|L_{n}(z ; f)-\sum_{j=0}^{l-1} s_{n, j}(z ; f)\right|\right\}^{1 / n} \leq \frac{\tau}{\rho^{l+1}} .
$$

Further, the result of (2.28) is best possible in the sense that there is some $\hat{f} \in A_{\rho}$ and some $\hat{z}$ with $|\hat{z}|=\rho^{l+1}$ for which the sequence

$$
\left\{L_{n}(\hat{z}: \hat{f})-\sum_{i=0}^{l-1} s_{n, j}(\hat{z}: \hat{f})\right\}_{n=1}^{\infty}
$$

does not tend to zero as $n \rightarrow \infty$.

To deduce an analogue of Theorem B for Theorem 1, we take the sum of the $p$ th terms corresponding to the summation index $s=p-1$ in each of the three kernels in (2.2) to form the kernel

$$
\begin{aligned}
\tilde{K}_{p}(t, z):= & \frac{z^{r m}\left(t^{n+1}-z^{n+1}\right)}{t^{(r+p) m+n+1}}+\frac{z^{n+1}\left(t^{m}-z^{m}\right) \gamma_{p-1}\left(z^{m}\right)}{t^{(r+p) m+n+1}} \\
& -\frac{\left(t^{m}-z^{m}\right) \gamma_{p-1}\left(z^{m}\right)}{t^{(r+1+p) m}}
\end{aligned}
$$

for all $p=1,2, \ldots$. This kernel is then used to define

$$
\text { (2.31) } \tilde{S}_{r m+n, p}(z ; f):=\frac{1}{2 \pi i} \int_{\Gamma} \frac{f(t) \tilde{K}_{p}(t, z) d t}{(t-z)}, \text { for } p=1,2, \ldots
$$

As $\gamma_{p-1}\left(z^{m}\right) \in \pi_{(r-1) m}$, it is evident that $\tilde{S}_{r m+n, p}(z) \in \pi_{r m+n}$ for each $p=1,2, \ldots$, and these polynomials $\tilde{S}_{r m+n, p}(z)$ form the analogs of the polynomials of (2.27). It is then convenient to set

$$
S_{r m+n, l}(z ; f):=s_{r m+n}(z ; f)+\sum_{p=1}^{l-1} \tilde{S}_{r m+n, p}(z ; f),
$$

$$
l=1,2, \ldots,
$$


with the convention that $\sum_{p=1}^{0} \equiv 0$. With these polynomials, we state the following result which gives Theorem 1 as the special case $l=1$.

THEOREM 2. For each $f \in A_{\rho}$, and for each nonnegative integer $r$, let the polynomials $P_{r m+n, l}(z ; f)$ and $S_{r m+n, l}(z ; f)$ be defined as in (1.5) and (2.32). With $m=n q+c$, where $q$ and $c$ are any fixed positive integers, and for each integer $l$, there holds

$$
\begin{aligned}
& \lim _{n \rightarrow \infty}\left\{\boldsymbol{P}_{r m+n}(z ; f)-S_{r m+n, l}(z ; f)\right\}=0, \\
& \qquad \text { for all }|z|<\boldsymbol{\rho}^{1+q /(1+r q),}
\end{aligned}
$$

the convergence being uniform and geometric on any closed subset of $|z|<\rho^{1+q /(1+r q)}$. More precisely, for any $\tau$ with $\rho \leq \tau<\infty$, there holds

$$
\varlimsup_{n \rightarrow \infty}\left\{\max _{|z| \leq \tau}\left|P_{r m+n}(z ; f)-S_{r m+n, l}(z ; f)\right|\right\}^{1 / n} \leq \frac{\tau^{r q+1}}{\rho^{(r+l) q+1}} .
$$

Further, the result of (2.33) is best possible in the sense that there is some $\hat{f} \in A_{\rho}$ and some $\hat{z}$ with $|\hat{z}|=\rho^{1+q / 1+r q)}$ for which the sequence

$$
\left\{P_{r m+n}(\hat{z} ; \hat{f})-S_{r m+n, l}(\hat{z} ; \hat{f})\right\}_{n=1}^{\infty}
$$

does not tend to zero as $n \rightarrow \infty$.

We omit the proof of this theorem, as it follows along the lines of the proof of Theorem 1 .

We conclude this section with the following remarks. Now, Theorem B makes no statement concerning the behavior of the sequence

$$
\left\{L_{n}(z ; f)-\sum_{j=0}^{l-1} s_{n, j}(z ; f)\right\}_{n=1}^{\infty}
$$

in $|z|>\rho^{l+1}$. To rectify this, Saff and Varga [5] have recently established

THEOREM C ([5]). For each $f \in A_{\rho}$ and for each positive integer $l$, the sequence (2.35) can be bounded in at most l distinct points in $|z|>\rho^{l+1}$. This result is sharp in the sense that, given any $l$ distinct points $\left\{\eta_{k}\right\}_{k=1}^{l}$ in $|z|>\rho^{l+1}$, there is an $\hat{f} \in A_{\rho}$ for which

$$
\lim _{n \rightarrow \infty}\left\{L_{n}\left(\eta_{k} ; \hat{f}\right)-\sum_{j=0}^{l-1} s_{n, j}\left(\eta_{k} ; \hat{f}\right)\right\}=0 \quad \text { for all } k=1,2, \ldots, l .
$$

It is an open question if Theorem 2 admits a Theorem C-type extension in $|z|>\rho^{1+q /(1+r q)}$. 
3. Birkhoff interpolation. It is natural to consider the following more general form of Birkhoff interpolation to generalize the interpolation of (1.5), thereby leading us to a generalization of Theorem 1 .

To begin, consider any $r$ fixed distinct positive integers $\left\{v_{j}\right\}_{j=1}^{r}$ satisfying

$$
\left(v_{0}:=\right) 0<v_{1}<v_{2}<\cdots<v_{r},
$$

and let $\bar{v}$ denote the vector $\left(0, v_{1}, \ldots, v_{r-1}\right)$. For any $f(z)$ in $A_{\rho}$, let $\pi_{N}(\bar{v} ; f)$ denote the class of polynomials, in $\pi_{N}$ where $N:=r m+n$, which satisfy

$$
\begin{aligned}
& Q_{N^{\prime}}^{(v)}\left(\omega^{k}\right)=f^{(v)}\left(\omega^{k}\right), \\
& \qquad \text { for all } k=0,1, \ldots, m-1 ; j=0,1, \ldots, r-1,
\end{aligned}
$$

where $\omega$ is any primitive $m$ th root of unity.

Now, the (weak) Pólya condition:

$$
v_{j} \leq j m \text { for all } j=0,1,2, \ldots, r-1,
$$

is clearly satisfied for all positive integers $m$ sufficiently large. Thus, as condition (3.3) is both necessary and sufficient (cf. [1]) to find a polynomial in $\pi_{r m-1}$ satisfying (3.2), then the set $\pi_{N}(\bar{v} ; f)$ is evidently nonempty for all $m$ sufficiently large.

As before, consider all positive integers $m$ of the form $m=q n+c$ where $q$ and $c$ are fixed positive integers. As the set $\pi_{N}(\bar{v} ; f)$ is nonempty for all $n$ sufficiently large, let $P_{N}(z ; f)$ be that element of $\pi_{N}(\bar{v} ; f)$ such that

$$
\begin{aligned}
& \sum_{k=0}^{m-1}\left|P_{N}^{\left(v_{r}\right)}\left(\omega^{k}\right)-f^{\left(v_{r}\right)}\left(\omega^{k}\right)\right|^{2} \\
& =\min _{Q_{N} \in \pi_{N}(\bar{v} ; f)} \sum_{k=0}^{m-1}\left|Q_{N}^{\left(v_{r}\right)}\left(\omega^{k}\right)-f^{\left(v_{r}\right)}\left(\omega^{k}\right)\right|^{2} .
\end{aligned}
$$

Again, the existence and uniqueness of $P_{N}(z ; f)$ is clear, and an explicit integral representation for $P_{N}(z ; f)$ can be derived, as was the analogous case in $\S 2$.

We state the following result which gives Theorem 1 as the special case when the integers $\left\{v_{j}\right\}_{j=1}^{r}$ are chosen to be $\{j\}_{j=1}^{r}$.

THEOREM 3. For each $f \in A_{\rho}$, and for any positive integers $\left\{v_{j}\right\}_{j=1}^{r}$ satisfying (3.1), let the polynomials $P_{N}(z ; f)$ and $s_{N}(z ; f)$ be as defined in 
(3.4) and (1.1). With $m=n q+c$, where $q$ and $c$ are any fixed positive integers, there holds

$$
\lim _{n \rightarrow \infty}\left\{P_{N}(z ; f)-s_{N}(z ; f)\right\}=0, \text { for all }|z|<\boldsymbol{\rho}^{1+q /(1+r q)},
$$

the convergence being uniform and geometric on any closed subset of $|z|<\rho^{1+q /(1+r q)}$. More precisely, for any $\tau$ with $\rho \leq \tau<\infty$, there holds

$$
\varlimsup_{n \rightarrow \infty}\left\{\max _{|z| \leq \tau}\left|P_{N}(z ; f)-s_{N}(z ; f)\right|\right\}^{1 / n} \leq \frac{\tau^{r q+1}}{\rho^{(r+1) q+1}} .
$$

Further, the result of (3.5) is best possible in the sense that there is some $\hat{f} \in A_{\rho}$ and some $\hat{z}$ with $|\hat{z}|=\rho^{1+q /(1+r q)}$ for which the sequence

$$
\left\{P_{N}(\hat{z}: \hat{f})-s_{N}(\hat{z}: \hat{f})\right\}_{n=1}^{\infty}
$$

does not tend to zero as $n \rightarrow \infty$.

The proof of Theorem 3, while depending on the results of Riemenschneider and Sharma [3], and Saxena, Sharma, and Ziegler [6], follows along the lines of the proof of Theorem 1, and is omitted.

As further open questions, we finally ask if there are Theorem B-type and Theorem C-type extensions of Theorem 3.

\section{REFERENCES}

[1] A. S. Cavaretta, Jr., A. Sharma, and R. S. Varga, Hermite-Birkhoff interpolation in the $n$-th roots of unity, Trans. Amer. Math. Soc., 259 (1980), 621-628.

[2] _ Interpolation in the roots of unity: an extension of a theorem of J. L. Walsh, Resultate der Mathematik, 3 (1981), 155-191.

[3] S. D. Riemenschneider and A. Sharma, Birkhoff interpolation at the n-th roots of unity; convergence, Canad. J. Math., 23 (1981), 362-371.

[4] T. J. Rivlin, On Walsh equiconvergence, J. Approximation Theory, 36 (1982), 334-345.

[5] E. B. Saff and R. S. Varga, A note on the sharpness of J. L. Walsh's Theorem and its extensions for interpolations in the roots of unity, Acta Math. Hung., 41 (1983), 371-377.

[6] R. B. Saxena, A. Sharma, and Z. Ziegler, Hermite-Birkhoff interpolation on roots of unity and Walsh equiconvergence, Linear Algebra and Appl., 52 / 53 (1983), 603-615.

[7] Richard S. Varga, Topics in Polynomial and Rational Interpolation and Approximation, Seminaire de Mathématiques Supérieures, University of Montreal Press, 1982.

[8] J. L. Walsh, Interpolation and Approximation by Rational Functions in the Complex Domain, Amer. Math. Soc. Colloquium Publications, Volumn XX, Providence, Rhode Island, fifth edition, 1969.

Received March 5, 1984. Research supported in part by the Air Force Office of Scientific Research, and the Department of Energy.

Kent State University

KENT, OH 44242 